DOI: https://doi.org/10.46296/yc.v4i7edesp.0060

\title{
VÍNCULO UNIVERSIDAD Y SOCIEDAD: SU IMPORTANCIA PARA AFRONTAR LOS CAMBIOS EN LA SOCIEDAD
}

\section{UNIVERSITY AND SOCIETY CONNECTING: ITS IMPORTANCE TO FACE CHANGES IN SOCIETY}

\author{
Cedeño-Rodríguez Luiggy Javier ${ }^{1 *}$; Rodríguez-Borges Ciaddy Gina ${ }^{2}$ \\ 1,2 Universidad Técnica de Manabí, UTM. Portoviejo, Ecuador. \\ *Correo: manumauce@gmail.com
}

\begin{abstract}
Resumen
Este trabajo se propone identificar oportunidades de mejora en la relación universidad con la sociedad, a los fines de desarrollar un mayor número de actividades de vinculación y extensión universitaria. Esta investigación tuvo objetivo identificar oportunidades de mejora que se presentan en el vínculo universidad con la sociedad en Ecuador. Se procedió a efectuar una revisión bibliográfica sistemática de diferentes enfoques sobre la vinculación o extensión, empleando la metodología Desk Research, se consideraron los criterios frecuentemente empleados para la valoración de esta importante misión de las Instituciones Educación Superior, evaluándose experiencias de universidades latinoamericanas y regionales. En este estudio fue posible determinar que los elementos que en términos pedagógicos, psicológicos y culturales son incidentes en la vinculación de la universidad con la sociedad. Siendo algunos de los elementos determinados; la asignación presupuestaria destinada a esta misión fundamental de las universidades, la ausencia de políticas claramente definidas en cuanto a las actividades que integran esta vinculación en cada facultad, la falta de continuidad en los proyectos implementados, que impiden la internalización de estos y la obtención de los frutos esperados en esta relación. Se proponen un conjunto de cambios entre los que se propone: fortalecer las consultorías de servicios a entes públicos y privados, efectuar un mayor vínculo con los egresados con una periodicidad definida, fomentar la educación continuidad mediante taller empleando la virtualidad para estudiantes, sociedad y docentes, además de expandir las actividades de prácticas preprofesionales a diferentes entes públicos y privados.
\end{abstract}

Palabras Claves: extensión universitaria, educación continua, prácticas profesionales, egresados universitarios, ayudantías académicas.

\begin{abstract}
This work aims to identify opportunities for improvement in the university relationship with society, to develop a greater number of university outreach and outreach activities. The objective of this research was to identify opportunities for improvement that arise in the university-society link in Ecuador. A systematic bibliographic review of different approaches to linkage or extension was carried out, using the Desk Research methodology, the criteria frequently used for the assessment of this important mission of Higher Education Institutions were considered, evaluating experiences of Latin American and regional universities. In this study it was possible to determine that the elements that in pedagogical, psychological, and cultural terms are incidents in the connection of the university with society. Being some of the elements determined; the budgetary allocation for this fundamental mission of the universities, the absence of clearly defined policies regarding the activities that make up this link in each faculty, the lack of continuity in the
\end{abstract}

Información del manuscrito:

Fecha de recepción: 09 de septiembre de 2020.

Fecha de aceptación: 11 de noviembre de 2020.

Fecha de publicación: 16 de noviembre de 2020. 
implemented projects, which prevent their internalization and obtaining of the expected fruits in this relationship. A set of changes is proposed, among which it is proposed: to strengthen consulting services to public and private entities, to make a greater bond with graduates with a defined periodicity, to promote continuity education through workshops using virtuality for students, society, and teachers, in addition to expanding pre-professional internship activities to different public and private entities.

Keywords: university extension, continuing education, professional practices, university graduates, academic assistantships.

\section{Introducción}

La presente investigación surge de la necesidad de estudiar la relación entre la Universidad y la Sociedad, la cual se desarrolla bajo procesos estructurados curricularmente y no curriculares, contemplando proyectos temporales, pero también, las vinculaciones permanentes dentro de los programas académicos, que comprometen un conjunto de proyectos. La relevancia de esta interacción entre las universidades y la sociedad y sus necesidades de perfeccionamiento, ha sido motivo de diversos estudios a los fines de lograr perfeccionar estos nexos simbióticos, para identificar los elementos de mejora en esta relación.

Se entiende por vinculación, al proceso integral que articula las funciones sustantivas de docencia, investigación y extensión de la cultura y los servicios de las
Instituciones Educación Superior (IES), para su interacción eficaz y eficiente con el entorno social, económico y tecnológico de la sociedad (Simbaña Cabrera, 2017). Esta relación también puede ser concebida, a través del desarrollo de acciones y proyectos de beneficio mutuo entre la universidad $y$ diferentes representantes de organizaciones públicas y privadas que conforma la comunidad de una región, lo que contribuyen a su posicionamiento y reconocimiento social de ambas.

Las relaciones entre la Universidad y la Sociedad se suceden de diferentes formas, En algunas oportunidades se desarrollan procesos continuos, tales como los de formación de alumnos y docentes, y en otros casos de formas puntuales en el tiempo. En el caso particular de las universidades públicas por su naturaleza social, 
estas direccionada mediante las políticas del Estado, a ser un ente trasformador de la sociedad, por lo que es preciso que destine parte de su presupuestos al desarrollo de estas actividades y a la necesidad de financiar diversos proyectos de intercambio (Plata, 2006). De allí que se pueda hablar de una perspectiva social, cuando la Universidad favorece la vinculación con sectores sociales vulnerables, impulsa la extensión solidaria e investiga para elevar la calidad de vida del conjunto de la población (Pérez-Rodríguez, RodríguezBorges, Rodríguez, \& Villacreses, 2020).

Entre las organizaciones que deben dirigir esfuerzo para aumentar la excelencia y eficiencia de sus funciones, se encuentran las universidades, planteándose mejorar sus servicios, para lo cual se han propuesto acciones diversas como: adecuar y actualizar estructuras, rediseñar planes de estudio, estimular programas de investigación, mejorar la gestión administrativa, capacitar a docentes e investigadores (Meza \& Bei, 1991). Estos cambios para la mejora de las IES, tiene repercusiones en la sociedad, y están relacionada con el articulo $\mathrm{N}^{\circ} 3$, literal " $\mathrm{f}$ " de la ley de Educación Superior Ecuatoriana, que plantea que las IES, dentro de sus objetivos y estrategias le corresponde: "realizar actividad de extensión, orientada a vincular su trabajo académico con todos los sectores de la sociedad, sirviéndose mediante programas de apoyos a través de consultorías, asesoría, investigaciones, capacitaciones $u$ otros medios" (Ley de Educación Superior, 2013)

Es necesario reforzar de forma continua el énfasis que debe darse en la relación entre la Universidad y sociedad, reconociendo que está relación permite plantear posibles soluciones a los problemas cotidianos de las comunidades, pero también proponer soluciones innovadoras, la cuales son de interés para ambas partes, siempre y cuando se mantenga un seguimiento a los procedimientos hasta alcanzar la solución sistemática de los mismos.

En virtud de todo lo antes mencionado, debe contemplarse como prioritarias las actividades a desarrollar en las universidades públicas con la comunidad, de 
formar que la vinculación no se establezca de forma puntual sino permanente, para poder preparar a la sociedad de la que forma parte, para afrontar factores, tales como: la globalización, el desarrollo e innovación tecnológica necesarios, la demandas de solución a problemas sociales y ambientales, las nuevas formas de organización del trabajo, la pérdida de peso del sector público, el fomento de emprendimientos, entre otros; van a requerir con mayor intensidad la aplicación y desarrollo de modelos de responsabilidad social (Pastore, 2019).

Teniendo en cuenta todos los aspectos antes señalados, es preciso profundizar en la identificar de las oportunidades de mejora mediante el desarrollo de investigaciones dirigidas a este relevante temario, para ello es preciso inicialmente efectuar una revisión de los criterios y planteamiento esgrimidos por diferentes autores que muestran relevancia de este tema a nivel mundial, que nos permitan deducir los problemas de esta relación y sus oportunidades de mejora, frente a los desafíos socioeconómicos que enfrentan las sociedades de América Latina, considerando las estrategias implementadas en la IES de la región, considerando la vinculación académica con las comunidades y las organizaciones socioeconómicas, políticas 0 culturales (Pastore, 2019).

Las IES en general tiene una marcada inclinación hacia la búsqueda del desarrollo tecnológico, para estar a tono con las exigencias del entorno social y dar respuesta a los estudiantes en su capacitación para afrontar las necesidades de las empresas que los contrataran como futuros profesionales (YañezFigueroa et al., 2017). Dicho proceso de apropiación tecnológica viene acompañada de procesos de innovación (Rodríguez -Borges, Pérez Rodríguez, Lituma Ramírez, \& Pérez Baltar, 2020), que tienen que ver con procesos educativos creativo y novedoso (Pérez Rodríguez, Rodríguez Borges, Pérez, \& Bowen, 2020), que utiliza nuevas dinámicas de interacción entre los estudiantes, docentes y contenidos educativos, creando redes de colaboración con otras universidades y centros de 
investigación, por lo que todo estos aspectos van marcando el tipo de vínculo hacia actividades primordialmente de investigación formativa (Davydova \& Dorozhkin, 2016).

En las IES, las acciones pedagógicas deben estar centradas en el estudiante, la innovación se relaciona con procesos de calidad educativa, con el desarrollo de competencias necesarias para que los alumnos se enfrenten a problemas reales de su contexto, considerando la globalización y con la administración efectiva de los recursos de las Universidades (Sievers, 2016).

El proceso de formación de los estudiantes por medio del trabajo de vinculación, facilita la relacionan mediante 2 vías de producción de conocimientos (Polaino \& Romillo, 2017), y complementa el modelo investigativo universitario de Humboldt (Henningsen et al., 2013) lo que se puede considerar como el simple hecho de aportar útilmente a la sociedad con los conocimientos obtenidos en la academia o a través de investigaciones combinadas con las diferentes habilidades de los involucrados para dar soluciones.
Es preciso evaluar, si además de las actividades ya descritas, existen otros elementos que puede ser incorporados como mejoras en la relación de vínculo universidad y sociedad, por lo que esta investigación, tiene como objetivo identificar las oportunidades de mejora que se presentan en el vínculo universidad con la sociedad en Ecuador.

\section{Materiales y métodos}

El trabajo realizado está enmarcado en una investigación de tipo exploratoria, basada en la revisión de evidencias, es decir empleando la metodología Desk Research, considerando la temática de vinculación con la sociedad efectuada por las IES en Ecuador y Latinoamérica, así como también. Se incluyó el término de "extensión universitaria", el cual es empleado en algunos países de américa latina, EE.UU y Europa.

El análisis cualitativo efectuado estuvo basado en la identificación de forma esquemática de los elementos que conforman el proceso de vinculación con la sociedad, en los procesos ya delimitados por algunas universidades Ecuatoriana, 
las cuales mediante investigaciones previas a determinados un conjunto de actividades bajo las cuales se agrupan el accionar principal para fomentar el vínculo con la sociedad, se analizó la información recopilada de fuentes primaria y secundarias, considerando bases de datos científicas, tales como: Google académico, sciencedirect, Redalyc, Scielo, entre otras.

Se consideraron como criterios de búsquedas para la inclusión y exclusión de artículos para su análisis, las palabras claves, el periodo de publicación de los artículos (últimos 5 años), entre otros elementos, como la calidad del artículo de acuerdo en función del contexto. Finalmente, a partir de los datos más relevantes encontrados y metadatos, se plantearon los señalamientos y conclusiones que condujeron al informe de resultados (Revelo-Sánchez, CollazosOrdóñez, \& Jiménez-Toledo, 2018).

\section{Desarrollo}

La relación entre universidad y sociedad se puede manifestar de distintas maneras ya sea a través de la transferencia de conocimiento, investigaciones, prácticas y tecnología, así como también la participación social en el gobierno de la universidad (Fernández Lamarra \& Pérez Centeno, 2016). Pero una de sus principales roles es el vínculo universidad con la sociedad, actualmente constituye uno de los factores más sensibles a la hora de valorar y apreciar la pertinencia de la universidad y su entorno (Polaino \& Romillo, 2017). Es desde el año 2009 que se fomentado de estas actividades, la cual se desarrolla esta interacción, para la cual debe tenerse en cuenta muchos factores, con diversos orígenes e intereses, que inciden y caracterizan la realidad que debe sin dudas cada día mejorada, para fortalecer este vínculo.

En la revisión efectuada se involucra también cambios de paradigmas en el tipo de conocimiento válido, en la necesidad de diálogos entre saberes académicos y saberes prácticos, 0 en los objetivos de la producción de conocimiento universitario, sus destinatarios y los modos en que es priorizada su generación y acceso (CRES, 2018). Distintas 
universidades de América Latina, han identificado una alianza clave con los movimientos y actores vinculados a la economía social y solidaria, con la finalidad de impulsar el desarrollo socioeconómico territorial con estrategias que respondan simultáneamente a problemáticas sociales, económicas y ambientales (Pastore, 2019).

Las funciones sustantivas de la universidad, tiene su punto de partida en la trasmisión del conocimiento, fundamentalmente a través de la docencia; la generación y aplicación del nuevo conocimiento a través de la investigación; finalmente la difusión y extensión del conocimiento, a través de la vinculación universidad-sociedad (De la Torre, 2013). El ámbito de acción en la relación universidad y sociedad, tiene una concepción más amplia que la que ha sido expresada en la figura 1, en ellas se plantean 4 macro actividades que permiten abordar las principales tareas definidas en la relación universidad sociedad.

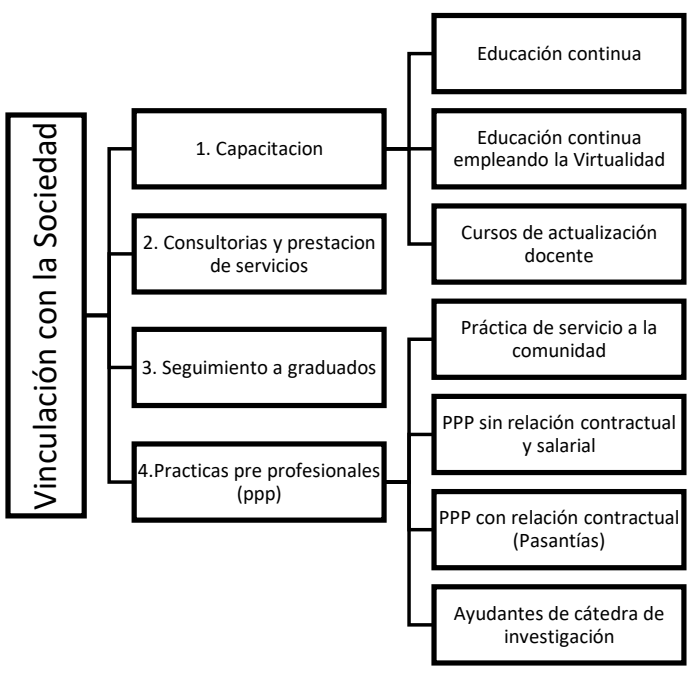

Figura.1. Diagrama del alcance del trabajo de Vinculación con la Sociedad, Adaptado del (Reglamento de Régimen Académico de la Universidad de Otavalo, 2016).

Elaboración: Autores.

La Universidad, sus docentes y estudiantes son agentes promotores de bienestar en sí mismos, a través de la trasmisión cultura y de saberes (lo cual, indudablemente, tendrá como efecto una mejora en las condiciones de vida) (Bordoli, 2010). Entre los elementos dispuestos por la Universidad, para la disposición del colectivo social, se encuentra el conocimiento generado por docentes y estudiantes (Henríquezl, Rodríguez, \& Quintero, 2020). Por medio de éstos y en diálogo constante con la comunidad deben articularse nuevas soluciones, positivas y creativas a los problemas emergentes del medio (Bordoli, 2010) 
La vinculación universidad y sociedad, beneficia a todos los que participan en ella, lo que genera desarrollo y modernización social, económica y tecnológica, disminuyendo las barreras entre el sector académico y productivo (Macías, Vélez \& Loor, 2017). Diversos análisis sobre la vinculación con la sociedad de la IES, ha sido abordados por autores donde destacando la necesidad establecer puente con el sectores empresarial (Ríos, 2005), tal es casos, de las universidades mexicanas, las cuales han celebrado diversos convenios de colaboración con el sector productivo; sin embargo, se manifiestan diversas debilidades y fallas, lo cual demuestra, que como en circunstancias similares, no basta con la pura voluntad hacerlo sino del seguimiento en la ejecución del plan.

En América Latina, existe un escaso seguimiento en la planificación universitaria, respecto a los fines y los resultados esperados en el conjunto de actividades desarrolladas en la vinculación con la sociedad, los entes públicos y privados aún o no establece un vínculo cercano con las IES para la cooperación entre ambas partes (Fernández Lamarra \& Pérez Centeno, 2016). Así mismo (Macías et al., 2017). La falta de claridad en el objetivo de la IES a la importancia de su aporte a la sociedad hace lento el proceso de vinculación, genera confusión en la relación con las actividades de extensión social y en la consultoría para la prestación de servicio.

Cabe agregar que (Naranjo, 2013), resalta que la vinculación de las IES con la sociedad, facilita la realización de cambios significativos en situaciones inequitativas, donde se demandan liderazgos transformadores. Las políticas de Extensión ubican a la Universidad en diálogo permanente con las organizaciones de la sociedad civil y del Estado, contribuyendo a partir del conocimiento y el pensamiento crítico, al estudio, diseño, formulación, monitoreo y evaluación de políticas públicas. (Menéndez, 2012) 


\subsection{Actividades de vinculación con la sociedad}

En las actividades de Vinculación con la sociedad, existe la oportunidad permanente de que las universidades mediante al análisis aporten soluciones a los conflictos del entorno social, para que desde una acción conjunta con otros entes se pueda contribuir con la transformación del mismos, empleando sus conocimientos, mediante las acciones de los estudiantes y los docentes sobre estos problemas, con liderazgo social y político hacia los territorios que demandan de una agenda de transformación y cambio (Fuerza ciudadana, 2016)

La vinculación universidad y sociedad, puede brindar respuestas concretas a las necesidades sociales, esto es puede permitir la recuperación e inserción válida de largo alcance de los sectores sociales, amplios y marginados históricamente en sus derechos, entre ellos la educación superior; lo que paulatinamente se ha resolviendo en Ecuador, México, Bolivia, entre otros países, mediante la creación de universidades indígenas, universidades en comunidades rurales, universidades inclusiva, entre otras acciones desarrolladas. (Cabeza et al., 2016).

Actividades de capacitación: las universidades, deben instar a la sociedad, hacia la construcción cognitiva de conocimientos para la mejora de practica que conduzcan a formas más eficiente para el desarrollo de diferentes trabajo y tareas, así como también para elevar la calidad de vida de la población en general, este es el rol sin precedente que debe ocupar la universidad, como espacio creador, pero también transformador de la sociedad. La Universidad surge como un espacio abierto al público y que abarca diversos ámbitos; incluidos la formación de los estudiantes, personal administrativo y docente, sino de la comunidad misma de la cual forma parte y es corresponsable de su desarrollo local. (Palacios, 2018)

Este proceso ha evolucionado en sus formas y alcances, para tener una perspectiva más precisa del desarrollo de la educación superior en Latinoamérica, es importante considerar el nivel de estudiantes que finalizan los estudios universitarios, los cuales cantidad 
que cada día asciende y se disminuye los niveles de deserción. (Fernández-Lamarra \& PérezCenteno, 2016)

La potencialidad educativa, así como la concesión de la extensión universitaria de forma bidireccional, puede permitir un encuentro de saberes, en correspondencia con la asunción ética de la transformación sociedad, que es un rol histórico de las universidades, que, si bien se halla condicionado las disposiciones de políticas y condiciones reales, las potencialidades son infinitas para la transformación, formación y modificación de su entorno. Debe partir de ubicar al otro y ubicarnos a nosotros mismos como sujetos con múltiples posibilidades para el trabajo comunitario de extensión y de impartición de formación educativa para una dimensión ética, ambiental, económica y social. (Bordoli, 2010).

La democratización de la educación implica no sólo asegurar el acceso a nuevos sectores de la población, sino también su ascenso a mejores condiciones de calidad de vida, en especial, para que las generaciones futuras cuenten con nuevos métodos de trabajo más eficientes capaces de participare en un mercado nacional y mundial, que amerita cada día una mayor calificación. (Fernández Lamarra \& Pérez Centeno, 2016)

La universidad tiene como desafío involucrarse en diferentes ámbitos de las comunidades para dar respuesta a los problemas, más allá del rol del componen de docencia e investigación que le atañe, está el de extensión universitaria (Dagnino, 2000) Así, la vinculación logrará ser la cara hacia el exterior de las tres funciones, cuestión que en la realidad no ocurre, y es poco probable que suceda, porque cada una de las otras tres funciones no va a delegar su contacto con la sociedad. (Dagnino, 2000)

De modo que la vinculación con la sociedad, constituye en la gestión tan relevante como la investigación y la docencia, para dar respuesta a las demandas que la sociedad exige (Aparicio, Xiomara De, Marco Chininin, Odalys Toledo, 2017) .Este rol de vinculación con la sociedad, se concibe como la tercera misión, a través de la actividad 
emprendedora de la Universidad, es decir, desde la visión de ésta como una institución básica para la transferencia de I+D 0 del conocimiento tecnocientífico. Configura un nuevo concepto de "capitalismo académico" y un nuevo papel de la Universidad saliendo de su tradicional "torre de marfil" (Etzkowitz, 2000; Schulte, 2004). La adecuada gestión de las patentes, modelos de utilidad y licencias, que se generan en una nueva relación entre la Universidad y la Sociedad o Empresa. (Chávez Alcívar et al., 2020)

Seguimiento a graduados: Las IES actualicen periódicamente los perfiles terminales de los programas que ofrecen para atender tanto las aspiraciones de los estudiantes como los requerimientos laborales y aseguren que el alumno aprenda lo previsto en los planes y programas de estudio, y refuercen los esquemas de evaluación de los aprendizajes para garantizar que los egresados cuenten con los conocimientos, competencias y valores éticos que corresponden a la profesión que eligieron. (Ramírez Domínguez, 2017)
Practicas preprofesionales (ppp):

el egreso de estudiantes como profesionales plantea la oportunidad de identificar nuevas demandas sociales respecto de la universidad, expresadas como requerimientos concretos que son percibidos como importantes tal es caso de la retroalimentación que estos puedan brindarle en el desempeño de sus funciones a la universidad para la adecuación de nuevos perfiles de egreso. (Michavila \& Calvo, 1998), Por lo que se propone fomentar de manera directa este intercambio como puente entre lo impartido y las nuevas necesidades de los sectores productivos, a continuación se presenta los roles que representa este intercambio:

\section{a) Función creadora:} relacionada con la creación, desarrollo, transmisión y crítica de la ciencia, la técnica y la cultura, considerando a la universidad como depositaria del conocimiento y responsable entonces de la gestión de este, especialmente en lo relacionado con las demandas de los agentes productivos respecto del conocimiento necesario para 
cubrir puestos de trabajo específicos.

\section{b) Preparación para el mundo} profesional: las exigencias de la sociedad en este sentido se enmarcan principalmente en la responsabilidad de la universidad para que la preparación profesional proporcionada a sus titulados les permita conseguir empleo, lo que se complementa con la real aplicación de los conocimientos y métodos científicos proporcionados por las instituciones de educación superior para el ejercicio de actividades profesionales.

c) Fomento del desarrollo de la sociedad: traducido en el apoyo científico y técnico al desarrollo cultural, social y económico de su entorno, a través de una colaboración estrecha con la industria y los agentes sociales, buscando orientar su quehacer especialmente investigador hacia los requerimientos de las actividades de la comunidad local y nacional, así como el compromiso con la formación permanente de las personas a lo largo de la vida.

\section{d) Transmisión de la cultura} universitaria: fomentando el debate, la exposición de ideas artísticas o estéticas, la comunicación de todos los campos del saber, democratizando y consensuando el conocimiento mediante el intercambio de ideas, el debate y a veces la confrontación, otorgando a la universidad de un contenido político, en el noble sentido del término, aquel que busca contribuir al pensamiento y el ejercicio democrático.

Estas demandas de la sociedad señalas anteriormente establecen a nuestro juicio el ámbito concreto en el que deben desarrollarse los enfoques y propuestas de responsabilidad social universitaria, debido a que fusionan con bastante precisión y claridad tanto los aspectos más esenciales del quehacer universitario como los principales elementos de las necesidades actuales de la sociedad del conocimiento (Gaete Quezada, 2017). 


\section{Consultorías y prestación de}

servicios: las universidades tienen vinculado su desarrollo futuro a los requerimientos y demandas de la respectiva sociedad en la que se insertan, o como plantean (Velthuis \& Pavón, 2008) "la evolución de las universidades no debe ser ajena a las tendencias del desarrollo social, cultural y económico de las regiones", identificando además algunas tendencias y cambios que condicionan el futuro de la educación superior: a) Globalización e internacionalización. (disolución de fronteras, relación con el tiempo, etc.), b) la creciente competencia entre universidades. (por la captación de alumnado, universidades corporativas), c) surgimiento de nuevas necesidades por parte de los ciudadanos y de la sociedad, d) las transformaciones demográficas donde la movilidad se efectué de forma permanente, así como las posibilidades de brindar servicio a nivel no solo regional, e) Nuevas posibilidades que ofrecen las tecnologías de la información y las comunicaciones para brindar consultorías, y apoyo en aspectos técnicos y legales

\section{Conclusiones}

Compartiendo los diferentes términos socializados en esta investigación, podemos decir: que la universidad contribuye a la solución de los problemas críticos que aquejan a la sociedad, y estos deben abordarse a través de la relación Universidad y sociedad, lo que facilitara la identificación de las necesidades, económicas, sociales y de políticas asistenciales, a través de las acciones de intercambio permanente y de cumplimiento de las políticas definidas en la universidad.

Entre las oportunidades de mejoras detectadas en el vínculo de relación universidad y sociedad se encuentran: seguir fomentando la capacitación continua e incluir el uso de herramientas virtuales para poder alcanzar a un mayor población estudiantil mediante la educación en línea, formación de docente de forma continua, intensificar la realización de proyectos universitarios para la capacitación de la sociedad por medios oficiales digitales, considerando que en una ciudad o en un país, es necesaria una estrategia de difusión cultural, es decir, expandir los canales de 
difusión, de vinculación o extensión. Así como también, establecer proyectos para la prestación de servicios de consultoría a entes públicos y privados que permita la difusión de nuevos conocimientos y de métodos de trabajo innovadores, además de mantener un estrecho vínculo con las practica preprofesionales y los egresados de carrera.

\section{Bibliografía}

Aparicio, D., Marco A Chininin, \& Odalys Toledo. (2017). El rol de la vinculación en la integración de las funciones sustantivas de la Universidad Metropolitana del Ecuador. Revista Universidad y Sociedad, 9(4), 37-43.

Bordoli, E. (2010). Aportes para pensar la extensión universitaria. Extensión en Obra, 13. https://documentos.una.ac.cr/ bitstream/handle/unadocs/81 30/Extension\%20en\%20obra _Universidad\%20de\%20la\%2 0Rep\%c3\%bablica.pdf?sequ ence $=2 \&$ is Allowed $=y \#$ page $=1$ 3

Cabeza, C., Bone., C., \& Quiñones., B. (2016). Reflexiones sobre la universidad y la sociedad. Dominio de las Ciencias, 2(2),
444-454.

https://dialnet.unirioja.es/servl et/articulo?codigo $=5761557$

Chávez- Alcívar, G., Moreira-Chica, M., \& Álava Barreiro, L. (2020). Vinculación con la sociedad: Un aporte al perfil profesional del trabajador social de la Universidad Técnica de Manabí. Contribuciones a las Ciencias Sociales.

https://www.eumed.net/rev/cc css/2020/07/vinculacionsociedad-aporte.html

Coronel, M., Borges, C., \& Quintero, H. (2020). Valoración de ámbitos formativos del docente universitario de la carrera educación en la universidad de los andestáchira. REFCalE: Revista Electrónica Formación y Calidad Educativa. ISSN 1390-9010, 7(3), 119-133. http://refcale.uleam.edu.ec/in dex.php/refcale/article/view/3 078

Dagnino, R. (2000). Vinculacionismo/Neovinculaci onismo. Racionalidad de la interacción universidadempresa en América latina (1955-1995).

Davydova, N., \& Dorozhkin, E. (2016). Management of a network interaction of educational organisations oriented to innovation 
development. Indian journal of science and technology, 9(29), 36-42. http://ncrao.rsvpu.ru/sites/def ault/files/docs/Management_o f_a_Network_Interaction_of_ Educational_Organisations_O riented_to_Innovation_Devel opment.pdf

De la Torre, M. (2013). La universidad que necesitamos. México: Juan Pablos Editor, SA.

https://repositorio.uc.cl/bitstre am/handle/11534/6409/0003 81896.pdf?sequence $=1$

Educación Superior, C. (2013). Reglamento de régimen académico. Gaceta oficial ecuatoriana.

https://www.gob.ec/sites/defa ult/files/regulations/202002/Reglamento\%20de\%20R $\%$ C3\%A9gimen\%20Acad\%C 3\%A9mico.pdf

Fernández Lamarra, N. R., \& Pérez Centeno, C. G. (2016). La educación superior latinoamericana en el inicio del nuevo siglo. Situación, principales problemas y perspectivas futuras. Revista Española de Educación Comparada, 27, 123-148. http://espacio.uned.es/fez/view/bibliu ned:reec-2016-numero275030

Gaete Quezada, R. (2017). Responsabilidad social universitaria: Una nueva mirada a la relación de la universidad con la sociedad desde la perspectiva de las partes interesadas. Un estudio de caso. http://beu.extension.unicen.e du.ar/xmlui/handle/12345678 9/232

Macías, C., Vélez, T., \& Loor, M. (2017). La Participación de la Comunidad Universitaria y su Vinculación con la Sociedad. Revista Científica Hallazgos21, 2(3). https://revistas.pucese.edu.ec /hallazgos21/article/view/192

Menéndez, G. (2012). Extensión y políticas públicas: La universidad en el centro del debate. + E: Revista de Extensión Universitaria, 2, 4046.

https://dialnet.unirioja.es/servl et/articulo? codigo $=7329605$

MEZA, L., \& Bei, G. (1991). La vinculación como estrategia de desarrollo en las Universidades Públicas. Education, 8. http://publicaciones.anuies.m x/pdfs/revista/Revista91_S2A 3ES.pdf

Michavila, F., \& Calvo, B. (1998). La universidad española hoy: Propuestas para una política universitaria. Síntesis Madrid.

Naranjo, G. (2013). Vinculación con la sociedad, construyendo sostenibilidad. 
Palacios, R. (2018). Cooperación universitaria, sociedad del conocimiento sustentabilidad social. ObIES, 2.

http://repository.udistrital.edu. co/handle/11349/19965

Pastore, R. E. (2019). Estrategias de vinculación universitaria para el fortalecimiento de la economía social y solidaria. Otra Economía, 12(21), 231247.

https://revistaotraeconomia.or g/index.php/otraeconomia/arti cle/view/14787

Pérez, J., Rodriguez, C., Rodriguez, M., \& Villacreses, C. (2020). Espacios maker: Herramienta motivacional para estudiantes de ingeniería eléctrica de la Universidad Técnica de Manabí, Ecuador. Espacios, 41(02). https://n9.cl/p4ivz

Pérez Rodriguez, J. A., Rodriguez Borges, C. G., Pérez, A. V., \& Bowen, C. A. (2020). Emulation of System as Strategy for Teaching of Mechanical System. International Journal of Psychosocial Rehabilitation, 24(2).

https://www.psychosocial.co m/article/PR200368/9917/

Plata, L. (2006). La vinculación Universidad-Sociedad desde una perspectiva social. Educación y educadores,
9(2),

79-93.

http://aquichan.unisabana.ed u.co/sabana/index.php/eye/ar ticle/view/664

Polaino, C., \& Romillo, A. de J. (2017). Vinculación con la Sociedad en la Universidad de Otavalo, Ecuador. Formación universitaria, 10(3), 21-30. https://doi.org/10.4067/S0718 $-50062017000300004$

Ramírez Domínguez, M. de J., Reséndiz Ortega, M., \& Reséndiz Ortega, M. E. (2017). Metodología De Seguimiento De Egresados Para Fortalecer La Vinculación De La Universidad Con La Sociedad (Methodology for Monitoring Graduates to Strengthen the Involvement of the University with Society). Revista global de Negocios, 5(3), 99-111. https://papers.ssrn.com/sol3/ papers.cfm?abstract_id $=2914$ 540

Revelo-Sánchez, O., CollazosOrdóñez, C. A., \& JiménezToledo, J. A. (2018). El trabajo colaborativo como estrategia didáctica para la enseñanza/aprendizaje de la programación: Una revisión sistemática de literatura. TecnoLógicas, 21(41), 115134. http://www.scielo.org.co/sciel o.php?pid=S0123- 
$77992018000100008 \&$ script $=$ sci_abstract\&tlng=en

Ríos, G. C. (2005). La vinculación universitaria: Ese oscuro objeto del deseo. 7(2), 13.

Rodríguez Borges, C., Pérez Rodríguez, J., Lituma Ramírez, E., \& Pérez Baltar, A. (2020). Software Development for Transformer Model Supporting Significant Learning Electrical Machines. International Journal of Psychosocial Rehabilitation, 24(2).

https://www.psychosocial.co m/article/PR200373/9933/

Sievers, J. (2016). Educational developer 2.0: How educational development leaders will need to develop themselves in the era of innovation. The Journal of Faculty Development, 30(2), 107-115.

Simbaña Cabrera, H. (2017). Las implicaciones pedagógicas de la vinculación con la sociedad en la formación docente de los estudiantes de la Facultad de Filosofía de la Universidad Central del Ecuador. https://dialnet.unirioja.es/servl et/dctes?codigo $=129770$

Velthuis, M., \& Pavón, L. (2008). Universidad Digital 2010. 528.
Yañez-Figueroa, J., RamírezMontoya, M., \& GarcíaPeñalvo, F. (2017). Vinculación universidadsociedad para la innovación educativa: Los casos de laboratorios ciudadanos. Innovación Educativa. Investigación, formación, vinculación y visibilidad, 201225.

https://knowledgesociety.usal. es/system/files/Ch9preprint\%283\%29.pdf 